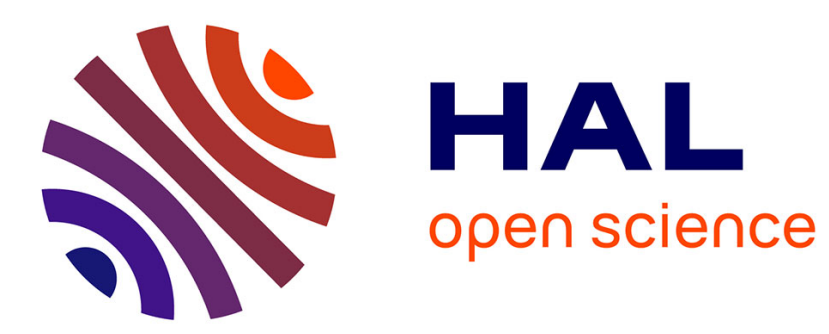

\title{
Evaluation of reaction fluxes in stationary and oscillating far-from-equilibrium biological systems
}

\author{
C Bianca, A Lemarchand
}

\section{To cite this version:}

C Bianca, A Lemarchand. Evaluation of reaction fluxes in stationary and oscillating far-fromequilibrium biological systems. Physica A: Statistical Mechanics and its Applications, 2015, 483, pp.1-16. 10.1016/j.physa.2015.06.012 . hal-01172704

\section{HAL Id: hal-01172704 https://hal.sorbonne-universite.fr/hal-01172704}

Submitted on 7 Jul 2015

HAL is a multi-disciplinary open access archive for the deposit and dissemination of scientific research documents, whether they are published or not. The documents may come from teaching and research institutions in France or abroad, or from public or private research centers.
L'archive ouverte pluridisciplinaire HAL, est destinée au dépôt et à la diffusion de documents scientifiques de niveau recherche, publiés ou non, émanant des établissements d'enseignement et de recherche français ou étrangers, des laboratoires publics ou privés. 


\title{
Evaluation of reaction fluxes in stationary and oscillating far-from-equilibrium biological systems
}

\author{
C. Bianca ${ }^{1,2}$ and A. Lemarchand ${ }^{* 1,2}$ \\ 1 Sorbonne Universités, UPMC Univ Paris 06, UMR 7600, \\ Laboratoire de Physique Théorique de la Matière Condensée, \\ 4, place Jussieu, case courrier 121, 75252 Paris cedex 05, France \\ ${ }^{2}$ CNRS, UMR 7600 LPTMC, Paris, France
}

May 5, 2015

\begin{abstract}
The complex spatio-temporal structures that appear in chemical and biological systems require far-from-equilibrium conditions which may lead to the circulation of reaction fluxes. We investigate how time asymmetry of cross-correlation functions of concentration fluctuations may be exploited to determine reaction fluxes at the cellular level. Using simulations of the master equation as a reference, we show that, far from a bifurcation, the Langevin approach provides a reliable tool to compute analytical expressions for time correlation functions. Biochemical mechanisms associated with bistability and oscillations issued from a Hopf bifurcation or a saddle-node infinite period bifurcation are considered. We show that the blind use of the simple relation obtained when assuming a linear deterministic dynamics often leads to a poor estimation of the value of the reaction flux and even of its sign.
\end{abstract}

Keywords: Time correlation functions, reaction flux, Hopf bifurcation, saddlenode bifurcation, Langevin equations, master equation

Corresponding author: Annie Lemarchand, e-mail: anle@lptmc.jussieu.fr

Other author: Carlo Bianca, e-mail: bianca@lptmc.jussieu.fr

Highlights:

- We design a protocol for determining a reaction flux in far-from-equilibrium systems.

- Time asymmetry of the correlation functions reveal departure from equilibrium.

- The fluctuations of the concentrations depend on the nonlinearities of dynamics.

- Time correlation functions are derived for bistable and oscillating systems. 


\section{Introduction}

The interplay between nonlinearity and departure from equilibrium is at the origin of complex spatio-temporal organization in chemical and biological systems $[1,2]$. The diversity of the structures developing at the cellular level is related to the nonlinearities of the dynamics, and organization occurs at the cost of energy or matter consumption. Sustained exchanges with the exterior, which drive the system of interest in far-from-equilibrium conditions, are necessary to the emergence of organized structures. Adenosine triphosphate (ATP) offers a well-known example of biochemical species whose concentration is regulated and that plays a crucial role in maintaining a biological system in far-from-equilibrium states. The evaluation of the departure from equilibrium in biology is therefore an essential task.

Recent progress in nonequilibrium statistical physics offers powerful tools to characterize far-from-equilibrium systems $[3,4,5,6,7,8,9,10,11]$. In particular, the fluctuation theorem $[4,5]$ states that, when considering a stochastic trajectory over a finite time $t$, the ratio between the probability that the time-averaged entropy production takes a value $V$ and the probability that it takes the opposite value exponentially depends on the product $V t$. Consequently, in a far-from-equilibrium system of sufficiently small size observed during a sufficiently small time, a negative entropy production can be a priori observed at such a mesosocpic scale, contrary to the prediction of the second law of thermodynamics, valid at the macroscopic scale. However, dealing with temperature in nonequilibrium conditions remains delicate, whereas isothermal chemical systems and the laws of chemical kinetics constitute a favorable framework to illustrate nonlinear behaviors specific to far-from-equilibrium

dynamical systems. Efficient fluorescent probes have been developed $[12,13]$ and fluorescence correlation spectroscopy (FCS) provides a sensitive detection method of concentration fluctuations, including in vivo investigations of small volumes [14]. Time asymmetry of the correlation functions of concentration fluctuations has been 
recently observed during endocytosis, a reactant-consuming process by which cells absorb molecules by engulfing them [15]. When the deterministic dynamics of such a far-from-equilibrium system is linear, the difference of cross-correlation functions of concentration fluctuations around a steady state has been shown to be universally related to the reaction flux circulating into the system [16]. Reaction flux refers to the notion of generalized flux introduced in thermodynamics of irreversible processes [17]. A thermodynamic flux is said to be conjugated to a thermodynamic force if their product gives the term of entropy production for the process of interest. In the case of a chemical system, the reaction flux is equal to the reaction rate and is conjugated to the affinity of the reaction. At equilibrium, all the chemical reactions obey detailed balance, the reaction fluxes vanish and time reversibility of the correlation functions of concentration fluctuations is recovered.

We propose to assess whether the determination of the cross-correlation functions can be used to evaluate the reaction flux in the biologically relevant cases of nonlinear deterministic dynamics. Specifically, we consider chemical mechanisms involving two variable concentrations and possessing either a stable nonequilibrium steady state or a stable limit cycle.

The paper is organized as follows. In the next Section, the method used to derive an analytical expression of the cross-correlation functions from Langevintype equations is given and the result obtained for linear dynamics is recalled [16, 18]. In Section 3, a nonlinear model of enzymatic catalysis is studied and the approximate expression of the cross-correlation functions deduced from the Langevin approach is compared to simulations of the chemical master equation, considered as the reference [18]. Then, chemical systems exhibiting oscillations of different natures are considered. We give the results obtained for the Brusselator model in the vicinity of a Hopf bifurcation [19] in Section 4. Another approach exploiting the normal form of the deterministic equations is used to evaluate the cross-correlation 
functions around the limit cycle. In Section 5, the limitations of the Langevin approach are pointed out in the case of large amplitude oscillations issued from a global bifurcation, the saddle-node infinite period bifurcation. The possibility to design a method for reaction flux evaluation from the cross-correlation functions in the absence of knowledge of the considered dynamics is discussed in Section 6 which concludes this paper.

\section{Cross-Correlation Functions Near a Steady State}

We consider a chemical mechanism involving two species $\mathrm{X}$ and $\mathrm{Y}$ of variable concentrations $X$ and $Y$. At the macroscopic scale, the rate laws are described by ordinary differential equations that are often nonlinear. The considered systems are supposed to possess at least a stable steady state $\left(X_{s}, Y_{s}\right)$. The biological system may be maintained far from equilibrium due to sustained exchanges of matter or energy with the exterior. The Langevin approach to chemical kinetics offers the simplest way to incorporate internal fluctuations into the description of small systems [20]. The Langevin equations are simply obtained by adding a Langevin force with zero mean to the deterministic equations. The variances of the Langevin forces compatible with the chemical master equation have well-known expressions depending on the rate constants and the concentrations $X$ and $Y$ [21, 22]. The linearized Langevin equations around the steady state $\left(X_{s}, Y_{s}\right)$ may be written as follows:

$$
\begin{aligned}
& \frac{d x}{d t}=a x+b y+\xi_{x}(t) \\
& \frac{d y}{d t}=c x+d y+\xi_{y}(t)
\end{aligned}
$$

where $x=X-X_{s}, y=Y-Y_{s}$ and the four real parameters $a, b, c$, and $d$ depend on the rate constants of the considered mechanism. The variances of the Langevin forces $\xi_{x}(t)$ and $\xi_{y}(t)$ are evaluated at the steady state $\left(X_{s}, Y_{s}\right)$ and denoted by

$$
\left\langle\xi_{x}(t) \xi_{x}\left(t^{\prime}\right)\right\rangle=F_{x x} \delta\left(t^{\prime}-t\right)
$$




$$
\begin{aligned}
& \left\langle\xi_{x}(t) \xi_{y}\left(t^{\prime}\right)\right\rangle=F_{x y} \delta\left(t^{\prime}-t\right) \\
& \left\langle\xi_{y}(t) \xi_{y}\left(t^{\prime}\right)\right\rangle=F_{y y} \delta\left(t^{\prime}-t\right)
\end{aligned}
$$

For discussing the stability of the steady state, we compute the eigenvalues $\lambda_{ \pm}$ of the matrix $\mathbf{M}$ associated with Eqs. $(1,2)$ :

$$
\mathbf{M}=\left(\begin{array}{ll}
a & b \\
c & d
\end{array}\right)
$$

which are solutions of the characteristic polynomial

$$
\lambda^{2}-\lambda(a+d)+a d-b c=0
$$

The solutions $\lambda_{ \pm}$can be real or complex numbers.

The new variables $x^{\prime}(t)$ and $y^{\prime}(t)$ in the basis of the eigenvectors are related to $x(t)$ and $y(t)$ according to

$$
\left(\begin{array}{l}
x(t) \\
y(t)
\end{array}\right)=\left(\begin{array}{cc}
P_{x x}=\frac{b}{\sqrt{b^{2}+\left(\lambda_{+}-a\right)^{2}}} & P_{x y}=\frac{b}{\sqrt{b^{2}+\left(\lambda_{-}-a\right)^{2}}} \\
P_{y x}=\frac{\lambda_{+}-a}{\sqrt{b^{2}+\left(\lambda_{+}-a\right)^{2}}} & P_{y y}=\frac{\lambda_{-}-a}{\sqrt{b^{2}+\left(\lambda_{-}-a\right)^{2}}}
\end{array}\right)\left(\begin{array}{c}
x^{\prime}(t) \\
y^{\prime}(t)
\end{array}\right)
$$

When the eigenvalues are real, $P_{x x}$ and $P_{y x}$ are the coordinates of the eigenvector associated with the eigenvalue $\lambda_{+}$, and $P_{x y}$ and $P_{y y}$ are the coordinates of the eigenvector associated with $\lambda_{-}$.

The correlation functions are easily determined for the new variables

$$
\begin{aligned}
& x^{\prime}(t)=x^{\prime}(0) e^{\lambda_{+} t}+\int_{0}^{t} e^{\lambda_{+}\left(t-t^{\prime}\right)}\left(P_{x x}^{-1} \xi_{x}\left(t^{\prime}\right)+P_{x y}^{-1} \xi_{y}\left(t^{\prime}\right)\right) \mathrm{d} t^{\prime} \\
& y^{\prime}(t)=y^{\prime}(0) e^{\lambda_{-} t}+\int_{0}^{t} e^{\lambda_{-}\left(t-t^{\prime}\right)}\left(P_{y x}^{-1} \xi_{x}\left(t^{\prime}\right)+P_{y y}^{-1} \xi_{y}\left(t^{\prime}\right)\right) \mathrm{d} t^{\prime}
\end{aligned}
$$

where $\mathbf{P}^{-1}$ is the inverse matrix of the change of basis matrix $\mathbf{P}$ defined in Eq. (8). Then, the change of basis matrix $\mathbf{P}$ is used again to compute the time correlation functions $\left\langle x(t) y\left(t^{\prime}\right)\right\rangle$ of the concentration fluctuations between times $t$ and $t^{\prime}$. In the long time limit, the correlation functions depend only on the time delay $\tau=t^{\prime}-t$ 
and the difference $I(\tau)$ of cross-correlation functions is given by [18]:

$$
\begin{aligned}
I(\tau) & =\langle x(0) y(\tau)\rangle-\langle x(\tau) y(0)\rangle \text { with } x=X-X_{s}, y=Y-Y_{s} \\
& =-\frac{\left(P_{y x} P_{y y} F_{x x}-\left(P_{x x} P_{y y}+P_{x y} P_{y x}\right) F_{x y}+P_{x x} P_{x y} F_{y y}\right)\left(e^{\lambda_{+}}-e^{\lambda_{-}}\right)}{\left(P_{x x} P_{y y}-P_{x y} P_{y x}\right)\left(\lambda_{+}+\lambda_{-}\right)}
\end{aligned}
$$

When the initial dynamics is linear, the difference of cross-correlation functions is proportional to the reaction flux $J$, which measures the departure from equilibrium $[16,18]$ :

$$
I(\tau)=J \frac{e^{\lambda_{+} \tau}-e^{\lambda_{-} \tau}}{\lambda_{+}-\lambda_{-}}
$$

At equilibrium, the reaction flux $J$ and the difference of cross-correlation functions $I(\tau)$ vanish. Conversely, when the system is maintained in a nonequilibrium steady state, the cross-correlation functions of concentration fluctuations are asymmetric under time reversal. The linearization of the Langevin equations around a steady state provides the same result as the exact solution of the master equation written for a linear dynamics [16]. The property expressed in Eq. (13) can be exploited to evaluate the reaction flux $J$ from the determination of the correlation functions of concentration fluctuations in a chemical or biological system using for example FCS for tagged fluorescent species. In the total absence of mechanistic and kinetic data on the system of interest, the reaction flux can be evaluated by the slope of $I(\tau)$ at time $\tau=0$, as suggested in reference [16]. If the typical chemical time scales of the system, i.e. the eigenvalues $\lambda_{ \pm}$, can be determined, then a better estimation of the reaction flux can be a priori obtained by using $J$ as a fitting parameter in Eq. (13). The method has been employed to study localized cellular events of the endocytic pathway using live-cell multichannel fluorescence imaging [15]. The cross-correlation functions of two species chosen among tagged clathrin, actin, and nexin have been assessed and the sign of the reaction flux has been deduced for an assumed linear dynamics. However, biological pathways often involve complex dynamical behaviors associated with specific nonlinearities. For example, regulation may be obtained 
with feedback loops which may induce oscillations, as observed in the well-known case of glycolysis $[1,23]$. In other cases, a bistable behavior may be observed and the system then switches between two different states as a threshold is crossed [24].

In the next Sections, our aim is to check the validity of Eq. (13) in the case of nonlinear chemical mechanisms. Three typical behaviors are investigated: Bistability [18], oscillations arising after a Hopf bifurcation [19], and oscillations resulting from a saddle-node infinite-period bifurcation.

\section{Bistable Enzymatic System}

Bistability is commonly encountered in biology. It is for example observed during mitosis, a phase of the cell cycle leading to the division of the mother cell into two identical daughter cells. The expression of total cyclin B1 controls the entrance into the mitotic phase. As the total cyclin B1 is increased, it is necessary to reach a larger threshold for the system to enter into mitosis than the threshold observed to leave the mitotic process and enter into interphase as the total cyclin B1 is decreased. A well-formed hysteresis loop has been recently observed in extracts of eggs of the frog xenopus when measuring the concentration of a maturation promoting factor, the cyclin B1/CDK1 (cyclin-dependent kinase) complex, as the concentration of the total cyclin B1 varies [24].

Inorder to make explicit the consequences of bistability on the relation between cross-correlation functions and reaction flux, we consider a simple model and use a modified version of the well-known Michaelis-Menten mechanism for enzymatic catalysis, in which the autocatalytic production of species $\mathrm{X}$ by the reverse reaction 1 has been introduced, leading to a possible bistable behavior [18]:

$$
3 \mathrm{X}+\mathrm{S} \underset{k_{-1}}{\stackrel{k_{+1}^{*}}{\rightleftharpoons}} \mathrm{Z}+2 \mathrm{X}
$$




$$
\begin{aligned}
& \mathrm{Z} \stackrel{k_{+2}}{\rightleftharpoons} \mathrm{Y} \\
& k_{-2} \\
& k_{+3} \\
& \mathrm{Y} \underset{k_{-3}^{*}}{\rightleftharpoons} \mathrm{X}+\mathrm{P}
\end{aligned}
$$

The concentrations of species $\mathrm{S}$ and $\mathrm{P}$ are supposed to be constant due to appropriate exchanges with the surrounding. The notation $k_{+1}=k_{+1}^{*} S$ and $k_{-3}=k_{-3}^{*} P$ for apparent rate constants is employed. The laws of chemical kinetics lead to the following deterministic equations for the two variables $X$ and $Y$ :

$$
\begin{aligned}
& \frac{d X}{d t}=-k_{+1} X^{3}+k_{-1} Z X^{2}-k_{-3} X+k_{+3} Y \\
& \frac{d Y}{d t}=k_{-3} X-k_{+3} Y+k_{+2} Z-k_{-2} Y
\end{aligned}
$$

where the variable $Z$ may be eliminated using the conservation relation $X+Y+Z=$ $C$, where $C$ is constant. Depending on the parameter values, the system may admit one or three stationary states. In what follows, we set the rate constants $k_{ \pm 1}, k_{ \pm 2}$, $k_{+3}$ at the values given in the caption of Fig. 1 and use $k_{-3}$ as a convenient control parameter. The steady values $X_{s}$ of the concentration of species $\mathrm{X}$ as $k_{-3}$ varies are given in Fig. 1. Bistability is observed for $k_{-3}$ in the range $[0.468,1.196]$.

In the absence of exchanges with the surroundings, the system would reach an equilibrium state and each reaction given in Eqs. (14-16) would obey detailed balance, i.e. the rate of each chemical process would vanish. When the system is sustained in the nonequilibrium steady state $\left(X_{s}, Y_{s}, Z_{s}\right)$, with $Z_{s}=C-X_{s}-Y_{s}$, the reaction flux $J$ measures the departure from detailed balance

$$
J=k_{+1}\left(X_{s}\right)^{3}-k_{-1} Z_{s}\left(X_{s}\right)^{2}=k_{+2} Z_{s}-k_{-2} Y_{s}=k_{+3} Y_{s}-k_{-3} X_{s}
$$

For the nonlinear enzymatic system, the four parameters $a, b, c$ and $d$ introduced in Eqs. $(1,2)$ are given by

$$
a=-3 k_{+1}\left(X_{s}\right)^{2}+k_{-1}\left(2\left(C-Y_{s}\right) X_{s}-3\left(X_{s}\right)^{2}\right)-k_{-3}
$$




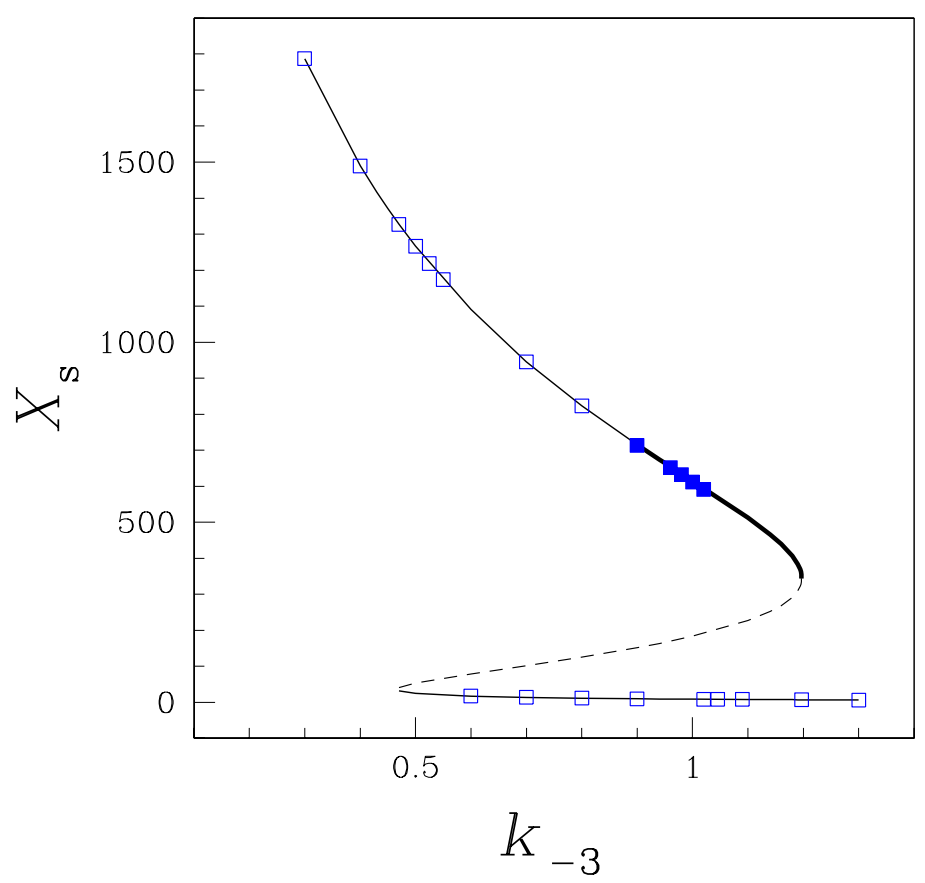

Figure 1. Bifurcation diagram associated with an autocatalytic enzymatic system. The lines give the stationary values $X_{s}$ of species $\mathrm{X}$ concentration, solutions of the nonlinear deterministic equations (Eqs. $(17,18)$ ), versus rate constant $k_{-3}$. The other parameters are fixed at $k_{+1}=10^{-6}, k_{-1}=4 \times 10^{-6}, k_{+2}=0.5, k_{-2}=0.1$, $k_{+3}=10^{-3}$. The solid lines are associated with the two stable branches of steady states. The thick line of the upper branch corresponds to focuses associated with complex eigenvalues and the thin lines to nodes associated with real eigenvalues. The dashed line is the unstable branch. The blue squares give the mean stable steady state values deduced from the solution of the master equation (Eq. (28)) for $N=10000$ particles and an ensemble average over $10^{7}$ realizations. The solid squares are associated with oscillating correlation functions and the open squares, with nonoscillating correlation functions.

$$
\begin{aligned}
b & =k_{+3}-k_{-1}\left(X_{s}\right)^{2} \\
c & =k_{-3}-k_{+2} \\
d & =-\left(k_{+2}+k_{-2}+k_{+3}\right)
\end{aligned}
$$

We employ an expression of the variances of the Langevin forces which is compatible with the chemical master equation [22]. When evaluated at the steady state, the 
variances become

$$
\begin{aligned}
& F_{x x}=k_{+1}\left(X_{s}\right)^{3}+k_{-1} Z_{s}\left(X_{s}\right)^{2}+k_{-3} X_{s}+k_{+3} Y_{s} \\
& F_{x y}=-\left(k_{-3} X_{s}+k_{+3} Y_{s}\right) \\
& F_{y y}=k_{+2} Z_{s}+k_{-2} Y_{s}+k_{-3} X_{s}+k_{+3} Y_{s}
\end{aligned}
$$

In the case of the autocatytic enzymatic system, the explicit calculation of the difference of cross-correlation functions using Eq. (12) leads to [18]:

$$
I(\tau)=J\left(1-\frac{4 k_{-3}}{\lambda_{+}+\lambda_{-}}\right) \frac{e^{\lambda_{+} \tau}-e^{\lambda_{-}}}{\lambda_{+}-\lambda_{-}}
$$

where the reaction flux $J$ is given by Eq. (19) and the eigenvalues $\lambda_{ \pm}$are solutions of Eq. (7) for the parameters $a, b, c$, and $d$ defined in Eqs. (20-23).

The validity of the linearized Langevin approach is checked by comparing the results given by Eq. (27) and a direct computation of the difference of cross-correlation functions from kinetic Monte Carlo simulations of the master equation for the probability of the numbers $N_{X}, N_{Y}$ and $N_{Z}$ of molecules of species $\mathrm{X}, \mathrm{Y}$ and Z, respectively $[20,21,25]$. The master equation associated with the mechanism described in Eqs. (14-16) reads:

$$
\begin{aligned}
& \frac{d P\left(N_{X}, N_{Y}, N_{Z}\right)}{d t}= \\
& =k_{+1}\left[\left(N_{X}+1\right) N_{X}\left(N_{X}-1\right) P\left(N_{X}+1, N_{Y}, N_{Z}-1\right)\right. \\
& \left.-N_{X}\left(N_{X}-1\right)\left(N_{X}-2\right) P\left(N_{X}, N_{Y}, N_{Z}\right)\right] \\
& +k_{-1}\left[\left(N_{Z}+1\right)\left(N_{X}-1\right)\left(N_{X}-2\right) P\left(N_{X}-1, N_{Y}, N_{Z}+1\right)\right. \\
& \left.-N_{Z} N_{X}\left(N_{X}-1\right) P\left(N_{X}, N_{Y}, N_{Z}\right)\right] \\
& +k_{+2}\left[\left(N_{Z}+1\right) P\left(N_{X}, N_{Y}-1, N_{Z}+1\right)-N_{Z} P\left(N_{X}, N_{Y}, N_{Z}\right)\right] \\
& +k_{-2}\left[\left(N_{Y}+1\right) P\left(N_{X}, N_{Y}+1, N_{Z}-1\right)-N_{Y} P\left(N_{X}, N_{Y}, N_{Z}\right)\right] \\
& +k_{+3}\left[\left(N_{Y}+1\right) P\left(N_{X}-1, N_{Y}+1, N_{Z}\right)-N_{Y} P\left(N_{X}, N_{Y}, N_{Z}\right)\right] \\
& +k_{-3}\left[\left(N_{X}+1\right) P\left(N_{X}+1, N_{Y}-1, N_{Z}\right)-N_{X} P\left(N_{X}, N_{Y}, N_{Z}\right)\right]
\end{aligned}
$$


with $N=N_{X}+N_{Y}+N_{Z}$. We use the algorithm introduced by Gillespie [26] to simulate the master equation. Each chemical process of the nonlinear mechanism has an assigned rate $r_{ \pm i}$, with $i=1,2,3$, equal to its transition probability in the master equation. For example, when the system contains $N_{X}$ particles of species X, the transition probability of the forward reaction 1 is given by $r_{+1}=k_{+1} N_{X}\left(N_{X}-1\right)\left(N_{X}-2\right)$. The total rate $r_{t o t}=\sum_{i=1}^{3} r_{ \pm i}$ is computed. Starting from a given state $\left(N_{X}, N_{Y}\right)$ and $N_{Z}=N-N_{X}-N_{Y}$, we randomly choose a process and a waiting time $\theta$ sampled from the distribution $r_{t o t} \exp \left(-r_{t o t} \theta\right)$. Time is increased by $\theta$, the new population values and the transition rates are updated according to the changes associated with the chosen reaction. Hence, a stochastic trajectory is generated in the phase space $\left(N_{X}, N_{Y}\right)$. The quantity $I(\tau)$ defined in Eq. (11) is computed using an ensemble average over a large number of stochastic trajectories.

The results of the three stochastic approaches are compared in Figs. 2, 3 for two values of the control parameter $k_{-3}$. The difference of cross-correlation functions $I(\tau)$ is calculated in the framework of the Langevin approaches, using Eq. in the hypothesis of linear deterministic dynamics and Eq. (27) for the nonlinear enzymatic system. The two Langevin approaches are compared to the simulations of the master equation, considered as a reference.

As shown in Fig. 2 for a sufficiently small value of $k_{-3}$, the three approaches are in good agreement. According to Fig. 3 obtained for a larger value of $k_{-3}$, the Langevin approach applied to nonlinear dynamics remains in good agreement with the results of the master equation and they both depart from the expression of $I(\tau)$ established for linear dynamics. The analytical result given in Eq. (27) can be considered as a good approximation of the correlation functions around the nonequilibrium steady state deduced from kinetic Monte Carlo simulations of the master equation. Some differences between the two approaches are discussed in 


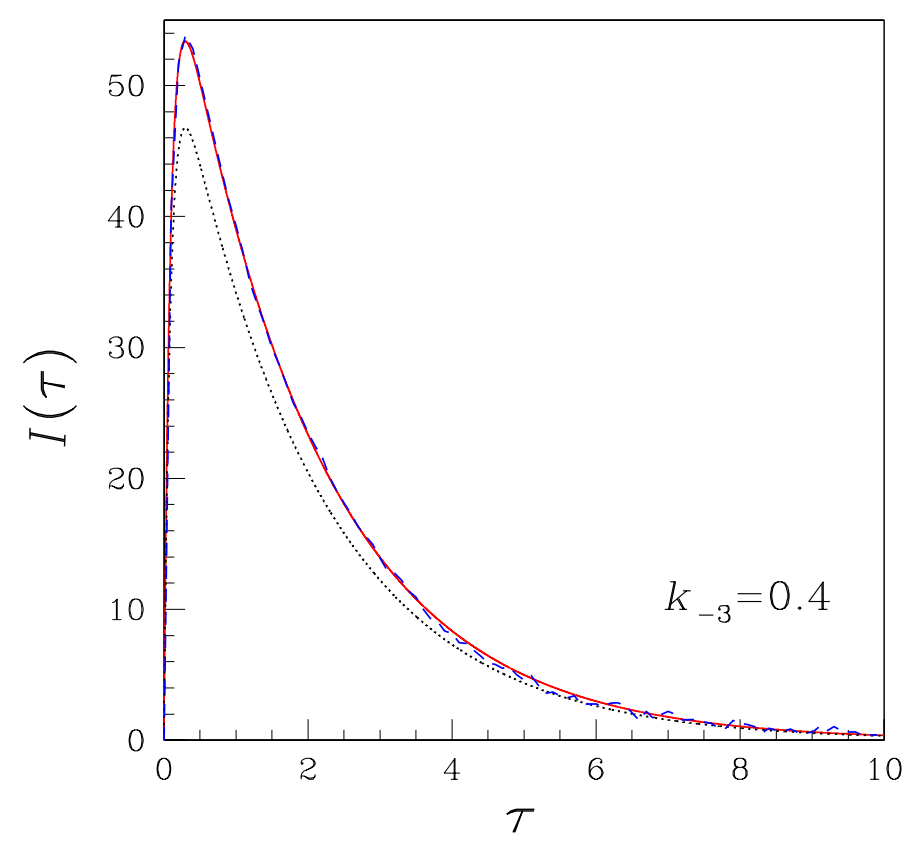

Figure 2. Enzymatic system. Difference of temporal cross-correlation functions $I(\tau)=\langle x(0) y(\tau)\rangle-\langle x(\tau) y(0)\rangle$ versus time lag $\tau$ for $k_{-3}=0.4$ in the domain of monostability associated with a high $X_{s}$ value. The black dotted line refers to the result of Eq. (13) deduced from Langevin approach to a system with assumed linear dynamics. The red solid line refers to the result of Eq. (27) deduced from Langevin approach to nonlinear dynamics with autocatalysis. The blue dashed line refers to the results of the direct simulation of the master equation (Eq. (28)) associated with nonlinear dynamics. The statistics is performed over $10^{7}$ stochastic trajectories. The other parameter values are given in the caption of Fig. 1.

reference [18] in the close vicinity of the bifurcation between monostability and bistability. The difference between the results of the Langevin approach to linear dynamics and the analogous approach applied to nonlinear dynamics increases as $k_{-3}$ increases, in agreement with the analytical expression of the correction term, $-\frac{4 k_{-3}}{\lambda_{+}+\lambda_{-}}$, appearing in Eq. (27). Typically, for the parameter values used to draw Fig. 1 and for $k_{-3} \simeq 1$, an underestimation of the reaction flux by a factor of 6 is obtained when computing $J$ from the slope of $I(\tau)$ at time $\tau=0$ when using Eq. (13) instead of Eq. (27). No divergence of the error is observed as the control parameter $k_{-3}$ crosses the bifurcation from monostability to bistability. In the next Section, we 
investigate the case of an oscillating system, also commonly encountered in biology and for which a less smooth behavior of the correlation functions is expected.

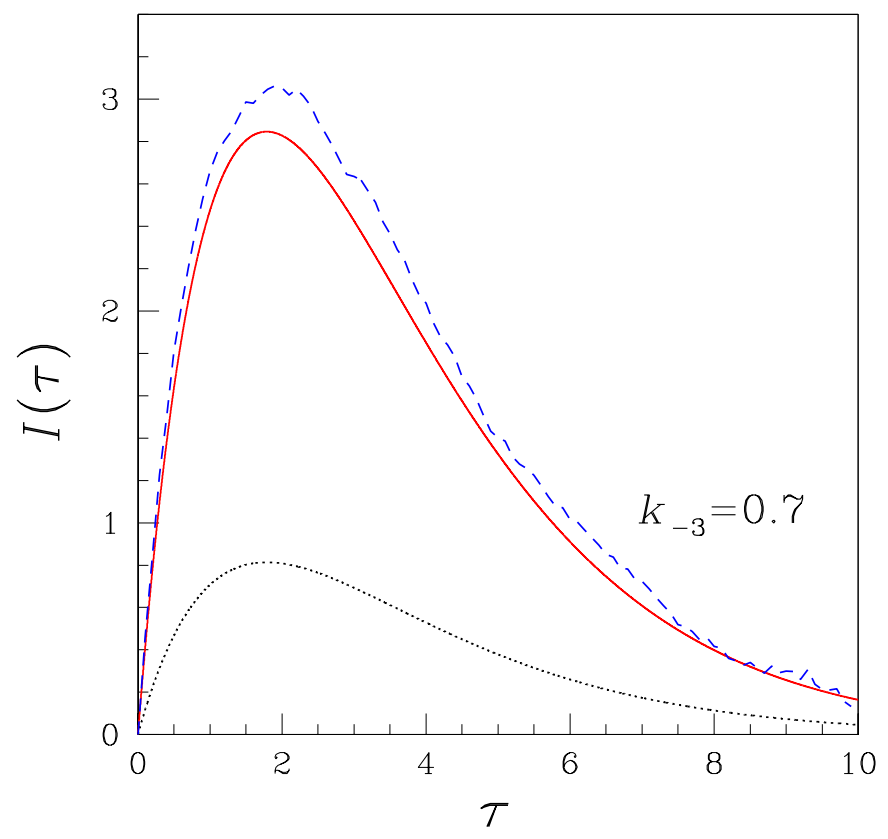

Figure 3. Enzymatic system. Difference of temporal cross-correlation functions $I(\tau)=\langle x(0) y(\tau)\rangle-\langle x(\tau) y(0)\rangle$ versus time lag $\tau$ for $k_{-3}=0.7$ in the bistable domain around the node associated with a lower $X_{s}$ value. See the caption of Fig. 2 to recover the meaning of the symbols and the parameter values.

\section{Oscillations associated with a Hopf Bifurcation}

One of the most studied examples of time periodic behaviors in biology is glycolysis [1]. Glycolysis is the metabolic pathway that converts glucose into pyruvate and the free energy released in the process is used to form the high energy compounds adenosine triphosphate (ATP) and reduced nicotinamide adenine dinucleotide (NADH). The emergence of time oscillations of NADH through a Hopf bifurcation has been observed in individual yeast cells as glucose or cyanide concentration is increased [23]. For the sake of simplicity and without loss of generality, we consider the reac- 
tion scheme associated with the Brusselator model in order to study the behavior of the correlation functions in the vicinity of a Hopf bifurcation [25, 27]:

$$
\begin{array}{rll}
\mathrm{A} & \stackrel{k_{+1}^{*}}{\rightleftharpoons} \mathrm{X} \\
& k_{-1} & \\
2 \mathrm{X}+\mathrm{Y} & \stackrel{k_{+2}}{\rightarrow} & \\
& & \\
& & \\
\mathrm{B}+\mathrm{X} & \stackrel{k_{+3}^{*}}{\rightarrow} & \mathrm{C}+\mathrm{Y}
\end{array}
$$

where the concentration of species $\mathrm{A}, \mathrm{B}$, and $\mathrm{C}$ is constant. The deterministic dynamics is governed by the following system of ordinary differential equations:

$$
\begin{aligned}
\frac{d X}{d t} & =k_{+1}-k_{-1} X+k_{+2} X^{2} Y-k_{+3} X \\
\frac{d Y}{d t} & =-k_{+2} X^{2} Y+k_{+3} X
\end{aligned}
$$

where we have set $k_{+1}=k_{+1}^{*} A$ and $k_{+3}=k_{+3}^{*} B$ for apparent rate constants.

The steady state $\left(X_{s}, Y_{s}\right)$ of the system reads:

$$
\begin{aligned}
X_{s} & =\frac{k_{+1}}{k_{-1}} \\
Y_{s} & =\frac{k_{+3} k_{-1}}{k_{+2} k_{+1}}
\end{aligned}
$$

In the next Subsections, we evaluate the difference of cross-correlation functions $I(\tau)$ defined in Eq. (11) using two different methods. We first apply Eq. (12), supposed to be valid in the vicinity of the steady state, and then evaluate the difference of cross-correlation functions around the limit cycle.

\subsection{Evaluation of the cross-correlation functions in the do- main of stability of the fixed point}

In the absence of the reverse steps associated with the forward reactions given in Eqs. $(30,31)$, i.e. for vanishing rate constants $k_{-2}=k_{-3}=0$, the Brusselator model intrinsically violates detailed balance and the reaction flux at steady state is different 
from zero. However, the first reaction given in Eq. (29) obeys detailed balance when $X=X_{s}$. When the system is in the steady state $\left(X_{s}, Y_{s}\right)$ given in Eq. $(34,35)$, the reaction flux can be written as follows:

$$
J=k_{+2} X_{s}^{2} Y_{s}=k_{+3} X_{s}
$$

The four parameters $a, b, c$ and $d$ of Eqs. (1,2), which define the linear stability operator $\mathbf{M}$ around the steady state, read:

$$
\begin{aligned}
a & =-k_{-1}+k_{+3} \\
b & =k_{+2}\left(X_{s}\right)^{2} \\
c & =-k_{+3} \\
d & =-k_{+2}\left(X_{s}\right)^{2}
\end{aligned}
$$

The solution of the characteristic polynomial defined in Eq. (7) are complex and written in the following form: $\lambda_{ \pm}=\alpha \pm i \beta$ where

$$
\begin{array}{r}
\alpha=\frac{k_{+3}-\left(k_{-1}+k_{+2}\left(X_{s}\right)^{2}\right)}{2} \\
\beta=\frac{1}{2} \sqrt{4 k_{-1} k_{+2}\left(X_{s}\right)^{2}-\alpha^{2}}
\end{array}
$$

A Hopf bifurcation occurs for $\alpha=0$, i.e. for

$$
k_{+3}=k_{-1}+k_{+2}\left(X_{s}\right)^{2}
$$

where $X_{s}$ is given in Eq. (34). For $\alpha>0$, the focus $\left(X_{s}, Y_{s}\right)$ is unstable and the attractor of the system is a limit cycle associated with oscillations of period $2 \pi / \beta$. The bifurcation diagram associated with the Brusselator model is given in Fig. 4 .

The variances of the Langevin forces evaluated at the steady state are given by [22]:

$$
\begin{aligned}
& F_{x x}=k_{+1}+k_{-1} X_{s}+k_{+2} X_{s}^{2} Y_{s}+k_{+3} X_{s} \\
& F_{x y}=-\left(k_{+2} X_{s}^{2} Y_{s}+k_{+3} X_{s}\right) \\
& F_{y y}=k_{+2} X_{s}^{2} Y_{s}+k_{+3} X_{s}
\end{aligned}
$$




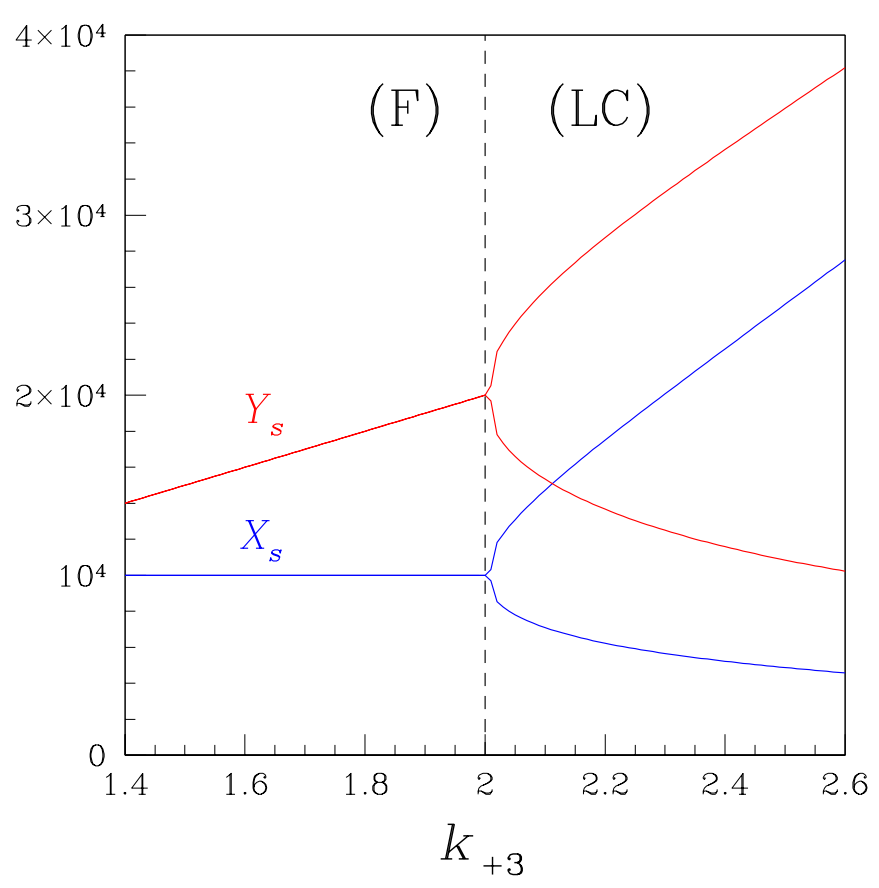

Figure 4. Bifurcation diagram associated with the Brusselator model. In the domain (F) where the focus is stable, variation of the coordinates $X_{s}$ (in blue) and $Y_{s}$ (in red) versus rate constant $k_{+3}$. In the domain (LC) where the limit cycle is stable, minimal and maximal values of the coordinates $X$ (in blue) and $Y$ (in red) on the limit cycle versus $k_{+3}$. The other parameters are fixed at $k_{+1}=10^{4}, k_{-1}=1$, and $k_{+2}=10^{-8}$. The critical value of the control parameter is $k_{+3}^{c}=2$.

Finally, Eq. (12) is used to find the expression of the difference of cross-correlation functions of concentration fluctuations around the focus [19]:

$$
I(\tau)=\frac{2 k_{-1}}{\alpha} J \exp (\alpha \tau) \frac{\sin (\beta \tau)}{\beta}
$$

Whereas the Langevin approach to the correlation functions given in Eq. (27) did not predict any singularity at the bifurcation points between monostable and bistable domains, the remarkable feature of Eq. (47) is the divergence of the amplitude of $I(\tau)$ at the Hopf bifurcation for which the real part, $\alpha$, of the eigenvalues vanishes. 


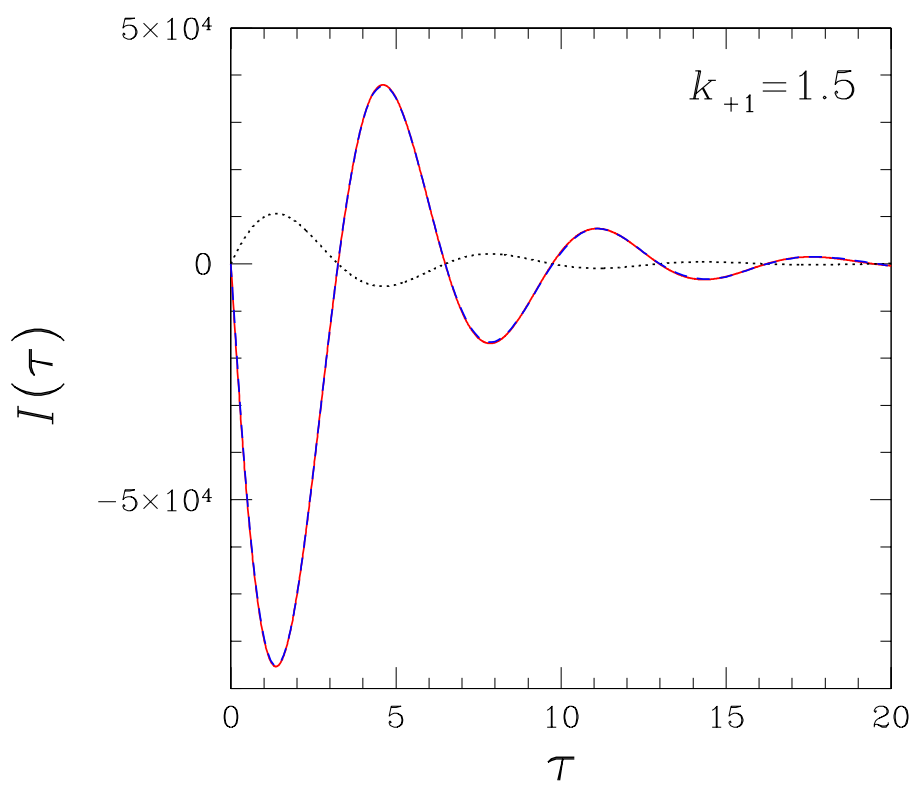

Figure 5. Brusselator model. Difference of temporal cross-correlation functions $I(\tau)=\langle x(0) y(\tau)\rangle-\langle x(\tau) y(0)\rangle$ versus time lag $\tau$ for $k_{+3}=1.5$ in the domain of stability of the focus $\left(X_{s}, Y_{s}\right)$. The other parameters are the same as in the caption of Fig. 4. The blue dashed line refers to the results of the direct simulation of the master equation. The statistics is performed over $10^{6}$ stochastic trajectories. The red solid line refers to the result of Eq. (47) deduced from Langevin approach. The black dotted line refers to the result of Eq. (13) valid when assuming linear deterministic dynamics for the same values of the reaction flux $J$ and the same eigenvalues $\lambda_{ \pm}$as for nonlinear dynamics.

\subsection{Evaluation of the cross-correlation functions in the do- main of stability of the limit cycle}

A different method relying on the determination of the normal form of the deterministic equations (Eq. (32,33)) can be used to evaluate the difference of crosscorrelation functions around the limit cycle [28, 29]. First, a linear change of basis is made so that the Jacobian around the steady state $\left(X_{s}, Y_{s}\right)$ is in the form

$$
\left(\begin{array}{cc}
\alpha & -\beta \\
\beta & \alpha
\end{array}\right)=\mathbf{Q}^{-1} \mathbf{M Q}
$$


where the elements of the matrix $\mathbf{Q}$, the departure from the fixed point, $(x=$ $\left.X-X_{s}, y=Y-Y_{s}\right)$, and the coordinates $\left(x^{\prime \prime}, y^{\prime \prime}\right)$ are related by

$$
\left(\begin{array}{l}
x \\
y
\end{array}\right)=\mathbf{Q}\left(\begin{array}{l}
x^{\prime \prime} \\
y^{\prime \prime}
\end{array}\right) \text {, with } \mathbf{Q}=\left(\begin{array}{cc}
k_{+2}\left(X_{s}\right)^{2} & 0 \\
-\alpha-k_{+2}\left(X_{s}\right)^{2} & -\beta
\end{array}\right)
$$

Using Eq. (49) and Eqs. $(32,33)$, we find that the dynamics of $\left(x^{\prime \prime}, y^{\prime \prime}\right)$ is governed by

$$
\begin{aligned}
& \frac{d x^{\prime \prime}}{d t}=\alpha x^{\prime \prime}-\beta y^{\prime \prime}+f\left(x^{\prime \prime}, y^{\prime \prime}\right) \\
& \frac{d y^{\prime \prime}}{d t}=\beta x^{\prime \prime}+\alpha y^{\prime \prime}+g\left(x^{\prime \prime}, y^{\prime \prime}\right)
\end{aligned}
$$

where the functions $f$ and $g$ are given by

$$
\begin{aligned}
f\left(x^{\prime \prime}, y^{\prime \prime}\right)= & k_{+2} X_{s}\left(k_{-1}-k_{+2}\left(X_{s}\right)^{2}\right) x^{\prime \prime 2}-2 k_{+2} X_{s} \beta x^{\prime \prime} y^{\prime \prime} \\
& -\left(k_{+2} X_{s}\right)^{2}\left(\alpha+k_{+2}\left(X_{s}\right)^{2}\right) x^{\prime \prime 3}-\left(k_{+2} X_{s}\right)^{2} \beta x^{\prime \prime 2} y^{\prime \prime} \\
g\left(x^{\prime \prime}, y^{\prime \prime}\right)= & -\frac{\alpha}{\beta} f\left(x^{\prime \prime}, y^{\prime \prime}\right)
\end{aligned}
$$

Then, near-identity tranformations, $X^{\prime \prime}=x^{\prime \prime}+h\left(x^{\prime \prime}, y^{\prime \prime}\right)$, where $h\left(x^{\prime \prime}, y^{\prime \prime}\right)$ is a nonlinear function, are used to eliminate nonresonant terms [30]. Finally, polar coordinates, such that $X^{\prime \prime}=R \cos (\theta), Y^{\prime \prime}=R \sin (\theta)$ are introduced, leading to

$$
\begin{aligned}
& \frac{d R}{d t}=R\left(\alpha-\gamma R^{2}\right) \\
& \frac{d \theta}{d t}=\beta-\delta R^{2}
\end{aligned}
$$

where the coefficients $\gamma$ and $\delta$ are evaluated at the bifurcation for $\alpha=0$ and at the steady state $x^{\prime \prime}=y^{\prime \prime}=0$. At leading order, we find [28, 29]:

$$
\begin{aligned}
\gamma= & -\frac{1}{16 \beta}\left(\left(f_{x^{\prime \prime} x^{\prime \prime} x^{\prime \prime}}+f_{x^{\prime \prime} y^{\prime \prime} y^{\prime \prime}}+g_{x^{\prime \prime} x^{\prime \prime} y^{\prime \prime}}+g_{y^{\prime \prime} y^{\prime \prime} y^{\prime \prime}}\right) \beta+f_{x^{\prime \prime} y^{\prime \prime}}\left(f_{x^{\prime \prime} x^{\prime \prime}}+f_{y^{\prime \prime} y^{\prime \prime}}\right)\right. \\
& \left.-g_{x^{\prime \prime} y^{\prime \prime}}\left(g_{x^{\prime \prime} x^{\prime \prime}}+g_{y^{\prime \prime} y^{\prime \prime}}\right)-f_{x^{\prime \prime} x^{\prime \prime}} g_{x^{\prime \prime} x^{\prime \prime}}+f_{y^{\prime \prime} y^{\prime \prime}} g_{y^{\prime \prime} y^{\prime \prime}}\right) \\
& =\frac{\left(k_{+2} X_{s}\right)^{2}}{8}\left(2 k_{-1}+k_{+2}\left(X_{s}\right)^{2}\right)
\end{aligned}
$$




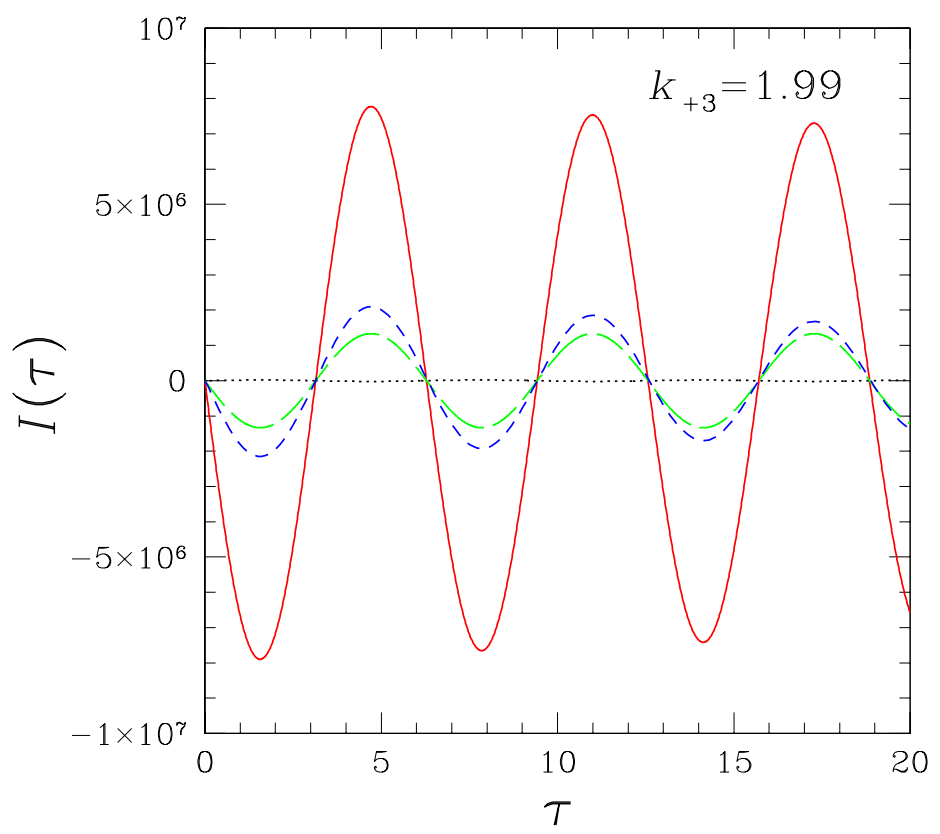

Figure 6. Brusselator model. Difference of temporal cross-correlation functions $I(\tau)=\langle x(0) y(\tau)\rangle-\langle x(\tau) y(0)\rangle$ versus time lag $\tau$ for $k_{+3}=1.99$, immediately before the Hopf bifurcation occurring at $k_{+3}^{c}=2$ for the same other parameters as in Fig. 4. The blue dashed line refers to the results of the direct simulation of the master equation. The statistics is performed over $10^{3}$ stochastic trajectories. The red solid line refers to the result of Eq. (47) deduced from Langevin approach. The green long-dashed line gives the results of Eq. (65) obtained when assuming the existence of a limit cycle. The black dotted line refers to the result of Eq. (13) valid when assuming linear deterministic dynamics for the same values of the reaction flux $J$ and the eigenvalues $\lambda_{ \pm}$as for nonlinear dynamics.

According to Eqs. $(54,55)$ and at leading order in $\alpha$, the dynamics on the limit cycle is given by

$$
\begin{aligned}
R_{L C} & =\sqrt{\frac{|\alpha|}{\gamma}} \\
\theta_{L C} & =\beta t
\end{aligned}
$$

or, equivalently, $X_{L C}^{\prime \prime}(t)=R_{L C} \cos (\beta t)$ and $Y_{L C}^{\prime \prime}(t)=R_{L C} \sin (\beta t)$. The inverse nearidentity transformations are used to switch from $\left(X_{L C}^{\prime \prime}, Y_{L C}^{\prime \prime}\right)$ to $\left(x_{L C}^{\prime \prime}, y_{L C}^{\prime \prime}\right)$ and the change-of-basis matrix $\mathbf{Q}$ given in Eq. (49) is used to switch from $\left(x_{L C}^{\prime \prime}, y_{L C}^{\prime \prime}\right)$ to 
$\left(x_{L C}, y_{L C}\right)$. At dominant order in $\alpha$, we get

$$
\left(\begin{array}{c}
x_{L C} \\
y_{L C}
\end{array}\right)=\left(\begin{array}{c}
X_{L C}-X_{s} \\
Y_{L C}-Y_{s}
\end{array}\right)=\mathbf{Q}\left(\begin{array}{c}
R_{L C} \cos (\beta t) \\
R_{L C} \sin (\beta t)
\end{array}\right)
$$

where $\left(X_{L C}, Y_{L C}\right)$ are the coordinates of a point on the limit cycle in the initial basis. Finally, the function $I_{L C}(\tau)$, to be compared with the difference of cross-correlation function $I(\tau)$, is defined on the limit cycle by

$$
\begin{aligned}
I_{L C}(\tau) & =x_{L C}(0) y_{L C}(\tau)-x_{L C}(\tau) y_{L C}(0) \\
& =\frac{-8 k_{+1}|\alpha|}{\sqrt{k_{+2} k_{-1}}\left(2 k_{-1}+k_{+2}\left(X_{s}\right)^{2}\right)} \sin (\beta \tau)
\end{aligned}
$$

where the steady concentration value $X_{s}$ is given in Eq. (34). The above equation is supposed to be valid only for positive values of the real part $\alpha$ of the eigenvalues of $\mathbf{M}$ around the fixed point. However, the introduction of absolute values gives the possibility to study the validity of the approach just above the bifurcation, for a still stable fixed point with $\alpha$ small but negative. On the limit cycle, the time averaged reaction flux can be defined as follows:

$$
J_{L C}=k_{+2}\left\langle X_{L C}^{2} Y_{L C}\right\rangle-k_{+3}\left\langle X_{L C}\right\rangle
$$

where $\langle$.$\rangle is here understood as a time average over the oscillation period \frac{2 \pi}{\beta}$. At leading order and at the bifurcation point, $\alpha=0$, the reaction flux $J_{L C}$ is evaluated at

$$
J_{L C}=-\left(k_{+2}\right)^{3}\left(X_{s}\right)^{5}\left(R_{L C}\right)^{2}
$$

where the radius $R_{L C}$ of the limit cycle is given in Eq. (58). Consequently, in the domain of stability of the limit cycle, the function $I_{L C}(\tau)$ given in Eq. (62) is related to the time averaged reaction flux $J_{L C}$ by

$$
I_{L C}(\tau)=\frac{J_{L C}}{k_{+2}} \sqrt{\frac{k_{-1}}{k_{+2}}}\left(\frac{k_{-1}}{k_{+1}}\right)^{2} \sin (\beta \tau)
$$

It is worth noting that the function $I_{L C}(\tau)$, deduced from a deterministic analysis, offers a first-order evaluation of the difference of cross-correlation functions of the 
concentration fluctuations in the vicinity of the limit cycle, whereas the derivation of Eq. (47) for $I(\tau)$ required a stochastic analysis of the fluctuations around the fixed point.

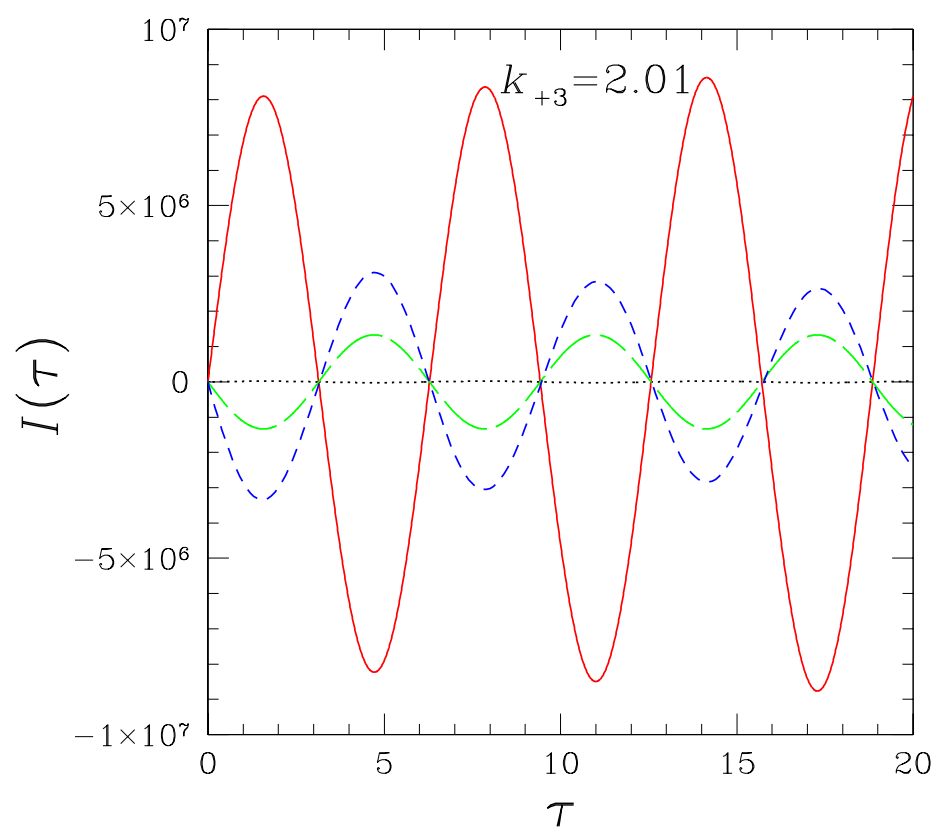

Figure 7. Brusselator model. Same caption as in Fig. 6 for $k_{+3}=2.01$, immediately after the Hopf bifurcation occurring at $k_{+3}^{c}=2$.

In the next Subsection, kinetic Monte Carlo simulations of the chemical master equation are used to check the validity of the difference of cross-correlation functions $I(\tau)$ and $I_{L C}(\tau)$ in the domain of stability of the focus and the domain of stability of the limit cycle, respectively.

\subsection{Comparison with simulations of the master equation}

The master equation for the probability of the number of particles $N_{X}$ and $N_{Y}$ of species $\mathrm{X}$ and $\mathrm{Y}$ is written for the chemical scheme given in Eqs. (29-31) [19] and numerically solved using the same lines as for the enzymatic system [26].

The two Langevin approaches to the cross-correlation functions and the numer- 


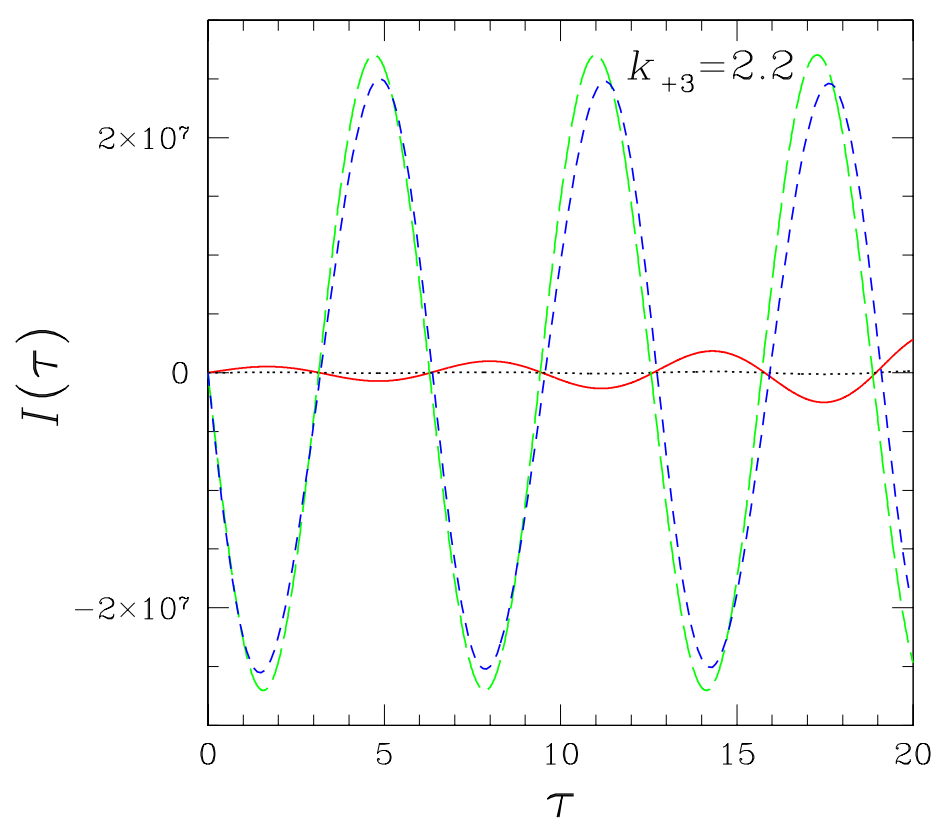

Figure 8. Brusselator model. Same caption as in Fig. 6 for $k_{+3}=2.2$, in the domain of stability of the limit cycle.

ical solutions of the master equation are compared in Figs. 5-9 for different values of the control parameter $k_{+3}$, above and beyond the bifurcation observed for the critical value $k_{+3}^{c}=2$. As shown in Fig. 5 sufficiently far from the Hopf bifurcation, the analytical result given in Eq. (47) following Lanvegin approach to nonlinear dynamics perfectly agrees with the simulations of the master equation, whereas the estimation provided by Eq. (13) for an assumed linear dynamics qualitatively differs and even leads to a false evaluation of the sign of the flux.

Figures 6 and 7 show the results of the three stochastic approaches around the fixed point and the deterministic approach on the limit cycle for parameter values very close to the Hopf bifurcation, just above and just beyond the bifurcation point, respectively. In these critical conditions, for which Eq. (47) predicts the divergence of $I(\tau)$, the Langevin approach to nonlinear dynamics does not correctly provide the amplitude of the oscillation and the three stochastic approaches disagree. While 
Eq. (47) overestimates the increase of the amplitude of the correlation functions in the vicinity of the bifurcation, Eq. (65) for $I_{L C}(\tau)$ correctly evaluates that the amplitude of the cross-correlation functions remains of the order of the product of the values taken by the concentrations on the limit cycle. According to Fig. 7, the evaluation of $I_{L C}(\tau)$ around the limit cycle compares well with the results of the master equation. In addition, the extension of the validity of Eq. (65) to the domain in which the limit cycle has still not appeared leads to a very good prediction of both the period $2 \pi / \beta$ and, more surprisingly, the amplitude, $\frac{-8 k_{+1}|\alpha|}{\sqrt{k_{+2} k_{-1}}\left(2 k_{-1}+k_{+2}\left(X_{s}\right)^{2}\right)}$, of the difference of cross-correlation functions, as shown in Fig. 6. Although obtained at leading order in the bifurcation parameter $\alpha$, Eq. (65) for $I_{L C}(\tau)$ provides a satisfying evaluation of the cross-correlation functions as $k_{+3}$ departs from the critical value $k_{+3}^{c}=2$ as observed in Fig. 8 for $k_{+3}=2.2$. However, as the control parameter reaches $k_{+3}=2.5$, the agreement between the predictions of Eq. (65) and the master equation becomes worse, as shown in Fig. 9. Indeed, the radius of the limit cycle $R_{L C}$ increases as $\sqrt{\alpha}$ and the first-order expansion in $\alpha$ becomes insufficient. Moreover, the shape of the limit cycle departs more from the circle as observed in the bifurcation diagram given in Fig. 4 and in the nonsymmetrical oscillations of the cross-correlation functions deduced from the master equation in Fig. 9.

Hence, the correlation functions around a focus in a system which possesses a Hopf bifurcation are extremely poorly evaluated when assuming a linear deterministic dynamics. The approximation would lead to a wrong information about the sign of the reaction flux and a mediocre evaluation of the absolute value departing from several orders of magnitude from the actual one. In contrast, the Langevin equations, linearized around the focus, give a good approximation of the correlation functions even if they overestimate the amplitude in the vicinity of the bifurcation. When the limit cycle is formed, the normal form of the deterministic equations 
gives access to an expression of the correlation functions. Instead of the non physical divergence of the correlations predicted by the local stochastic approach around the focus, the deterministic analysis around the limit cycle correctly accounts for the amplitude and period of the correlation functions, provided that the limit cycle remains sufficiently small.

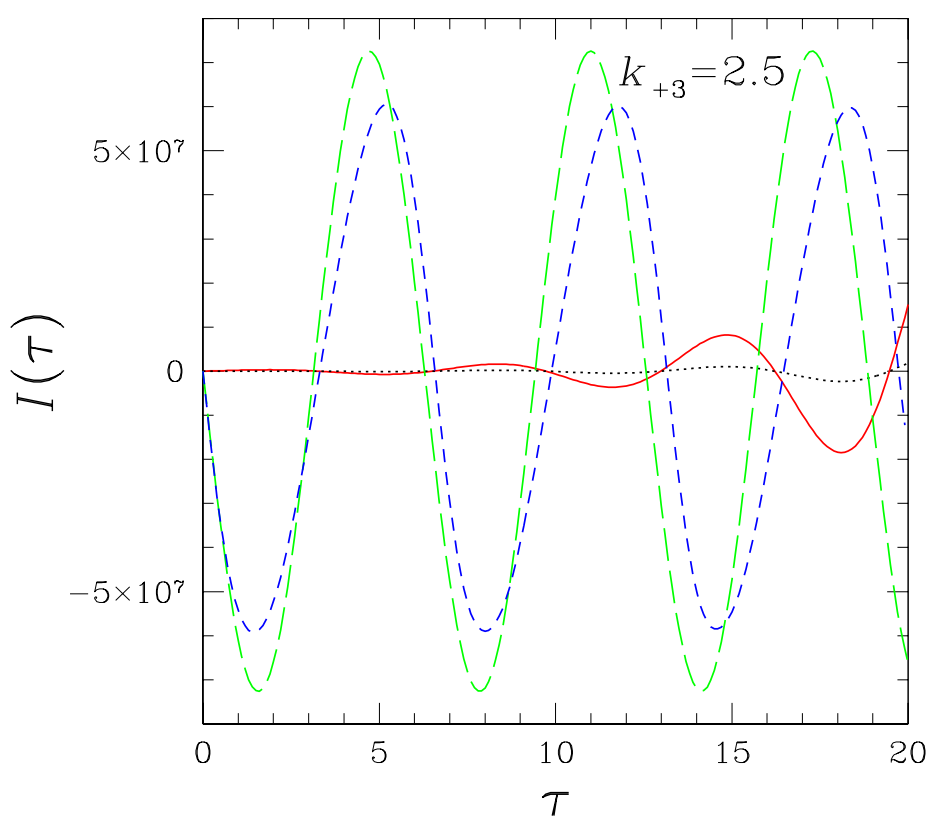

Figure 9. Brusselator model. Same caption as in Fig. 6 for $k_{+3}=2.5$, far in the domain of stability of the limit cycle.

In the next Section, we study the behavior of the difference of cross-correlation functions $I(\tau)$ as another type of bifurcation leading to oscillations arises, the saddlenode infinite-period bifurcation, for which a global analysis becomes rapidly necessary as the bifurcation approaches. 


\section{Chemical System Close to a Saddle-Node Infinite- Period Bifurcation}

The saddle-node infinite-period (SNIPER) bifurcation can be found in models of cell-cycle regulation, for example to account for a transition between interphase and mitosis $[31,32]$. Contrary to the (supercritical) Hopf bifurcation, which gives birth to a limit cycle with vanishing radius, the saddle-node infinite-period bifurcation leads to an already well-formed limit cycle from a homoclinic trajectory. A local analysis relying on an expansion in the departure from the bifurcation point is a priori of limited interest in the case of such a global bifurcation. In order to investigate the behavior of the correlation functions of the concentration fluctuations in the vicinity of a saddle-node infinite-period bifurcation, we use a slightly modified version of the two-variable chemical mechanism proposed by Erban et al. [33]:

$$
\begin{array}{rll}
\mathrm{A} \stackrel{k_{+1}^{*}}{\longrightarrow} \mathrm{Y} & \stackrel{k_{+2}}{\longrightarrow} \mathrm{X} \stackrel{k_{+3}}{\longrightarrow} \mathrm{B} \\
\mathrm{B}+2 \mathrm{X} & \stackrel{k_{+4}^{*}}{\rightleftharpoons} & \mathrm{X} \\
& \stackrel{k_{-4}}{\rightleftharpoons} \\
\mathrm{A}+\mathrm{X}+\mathrm{Y} & \stackrel{k_{+5}^{*}}{\longrightarrow} \mathrm{X}+2 \mathrm{Y} \\
& & \\
2 \mathrm{X}+\mathrm{Y} & \stackrel{k_{+6}}{\longrightarrow} & 2 \mathrm{X}+\mathrm{B}
\end{array}
$$

where we have introduced two species of constant concentrations $A$ and $B$. Apparent rate constants can be written as $k_{+1}=k_{+1}^{*} A, k_{+4}=k_{+4}^{*} B$, and $k_{+5}=k_{+5}^{*} A$. The macroscopic equations governing the dynamics of species $\mathrm{X}$ and $\mathrm{Y}$ are given by

$$
\begin{aligned}
& \frac{d X}{d t}=k_{+2} Y-k_{+3} X+k_{+4} X^{2}-k_{-4} X^{3} \\
& \frac{d Y}{d t}=k_{+1}-k_{+2} Y+k_{+5} X Y-k_{+6} X^{2} Y
\end{aligned}
$$

The stationary states are defined as the intersections of the nullclines $\frac{d X}{d t}=0$ and $\frac{d Y}{d t}=0$. Figures 10, 11 give two typical behaviors obtained as the control parameter 
$k_{+1}$ increases and crosses the critical value $k_{+1}^{c} \simeq 488$ associated with the saddle-node infinite-period bifurcation. As shown in Fig. 10 for $k_{+1}=440$ above the SNIPER bifurcation, the system has three steady states, an unstable node, a saddle and a stable node $\left(X_{s}, Y_{s}\right)$. At the bifurcation point, the node is absorbed by the saddle and a homoclinic trajectory is formed. Such an infinite period closed trajectory starts along the unstable eigendirection of the saddle and returns to the saddle point along the stable eigendirection.

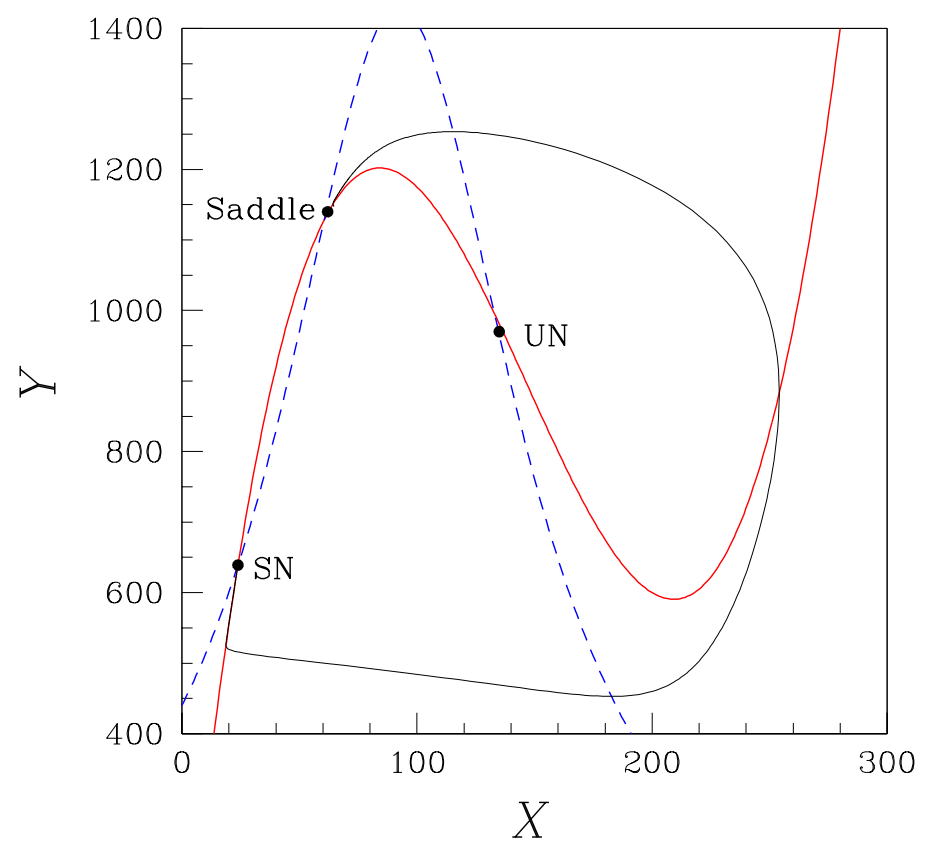

Figure 10. System with SNIPER bifurcation. Nullclines (blue dashed line and red solid line) obtained for $k_{+1}=440$, above the saddle-node infinite-period bifurcation. The other parameters are fixed at $k_{+2}=1, k_{+3}=33, k_{+4}=0.275, k_{-4}=0.000625$, $k_{+5}=0.015, k_{+6}=0.00008125$. The system possesses three steady states, a stable node (SN), a saddle and an unstable node (UN). The black solid line gives one trajectory connecting the saddle and the stable node.

In Fig. 11 for $k_{+1}=520$, beyond the saddle-node infinite period bifurcation, the system has a single unstable node (UN) surrounded by a stable limit cycle, directly formed from the homoclinic orbit after the disappearance of the saddle. 


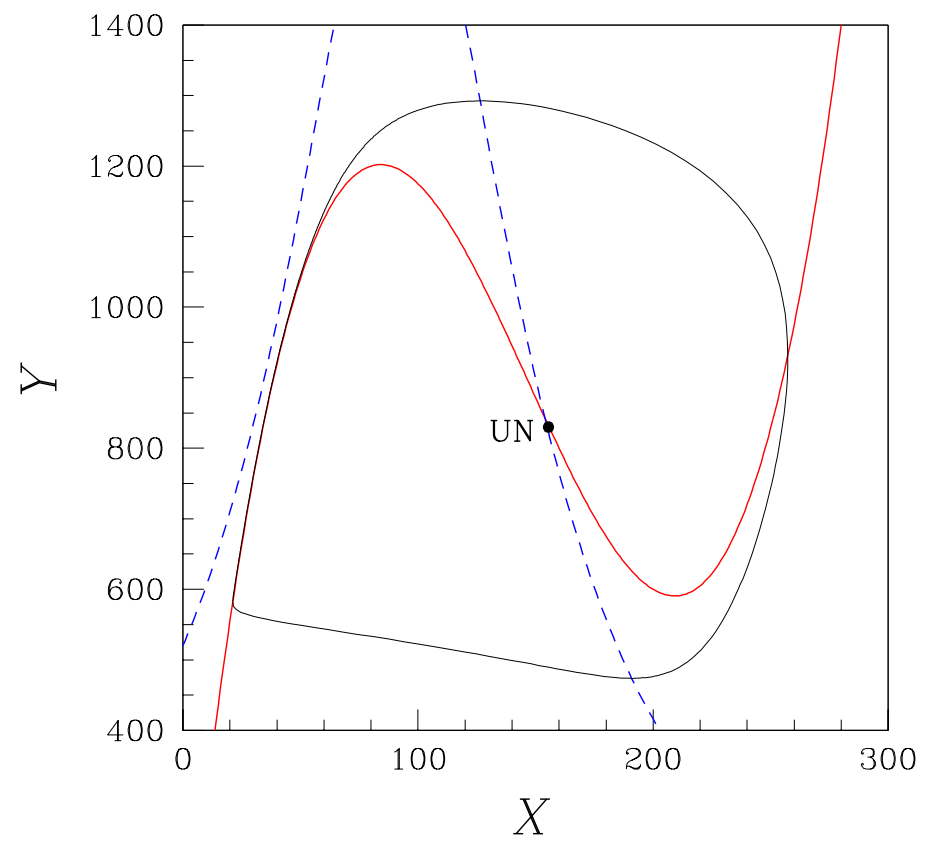

Figure 11. System with SNIPER bifurcation. Same caption as in Fig. 10 for $k_{+1}=520$, beyond the saddle-node infinite period bifurcation. The system possesses a single unstable node (UN) and a stable limit cycle (black solid line).

Above the SNIPER bifurcation, we define the reaction flux $J$ at the stable steady state $\left(X_{s}, Y_{s}\right)$ using the flux that the reservoir of species $\mathrm{A}$ has to inject into the system to counterbalance reactions 1 and 5 or, equivalently, the flux that the reservoir of species $\mathrm{B}$ has to remove from the system to counterbalance reactions $3,4,-4$ and 6 , in such a way that the concentrations of species A and B remain constant

$$
J=k_{+1}+k_{+5} X_{s} Y_{s}=k_{+3} X_{s}-k_{+4}\left(X_{s}\right)^{2}+k_{-4}\left(X_{s}\right)^{3}+k_{+6}\left(X_{s}\right)^{2} Y_{s}
$$

The four parameters $a, b, c$ and $d$ defining the linear operator in Eqs. $(1,2)$ are given by

$$
\begin{aligned}
a & =-k_{+3}+2 k_{+4} X_{s}-3 k_{-4}\left(X_{s}\right)^{2} \\
b & =k_{+2} \\
c & =k_{+5} Y_{s}-2 k_{+6} X_{s} Y_{s}
\end{aligned}
$$




$$
d=-k_{+2}+k_{+5} X_{s}-k_{+6}\left(X_{s}\right)^{2}
$$

The variances of the Langevin forces are

$$
\begin{aligned}
& F_{x x}=k_{+2} Y_{s}+k_{+3} X_{s}+k_{+4}\left(X_{s}\right)^{2}+k_{-4}\left(X_{s}\right)^{3} \\
& F_{x y}=-k_{+2} Y_{s} \\
& F_{y y}=k_{+1}+k_{+2} Y_{s}+k_{+5} X_{s} Y_{s}+k_{+6}\left(X_{s}\right)^{2} Y_{s}
\end{aligned}
$$

Then, Eqs. $(7,8)$ are used to compute the eigenvalues of the linear operator and the change of basis matrix. Finally, Eq. (12) gives the difference of cross-correlation function $I(\tau)$. For the sake of conciseness, we do not explicitly write the master equation associated with the SNIPER model, which can be easily written [21] and numerically solved using Gillespie algorithm [26].

The results are given in Figs. 12, 13 for $k_{+1}=440$ far from the SNIPER bifurcation and for $k_{+1}=487.6$, close to the critical value $k_{+1}^{c} \simeq 488$.

Exactly as in the case of the Hopf bifurcation, the analytical prediction of the Langevin approach to nonlinear dynamics (Eq. (12)) is validated by the simulations of the master equation as shown in Fig. 12, sufficiently far from the SNIPER bifurcation. As for the Hopf bifurcation, Eq. (13), obtained for linear dynamics, is not able to predict the sign of the flux, i.e. if the chemostats are solicitated to provide species A and absorb species B or the contrary. Close to the SNIPER bifurcation (see Fig. 13), the master equation leads to a deep minimum for the difference of cross-correlation functions whereas the Langevin approaches predict a maximum of small amplitude. Hence, for critical parameter values, the sign of the reaction flux is not correctly predicted by the Langevin approaches even when considering nonlinear dynamics. It is worth noting that the Langevin approaches are local, since the stochastic differential equations are linearized around the steady state even in the case of nonlinear dynamics. The limit cycle formed through the SNIPER bifurcation directly corresponds to a large excursion of the phase space. 


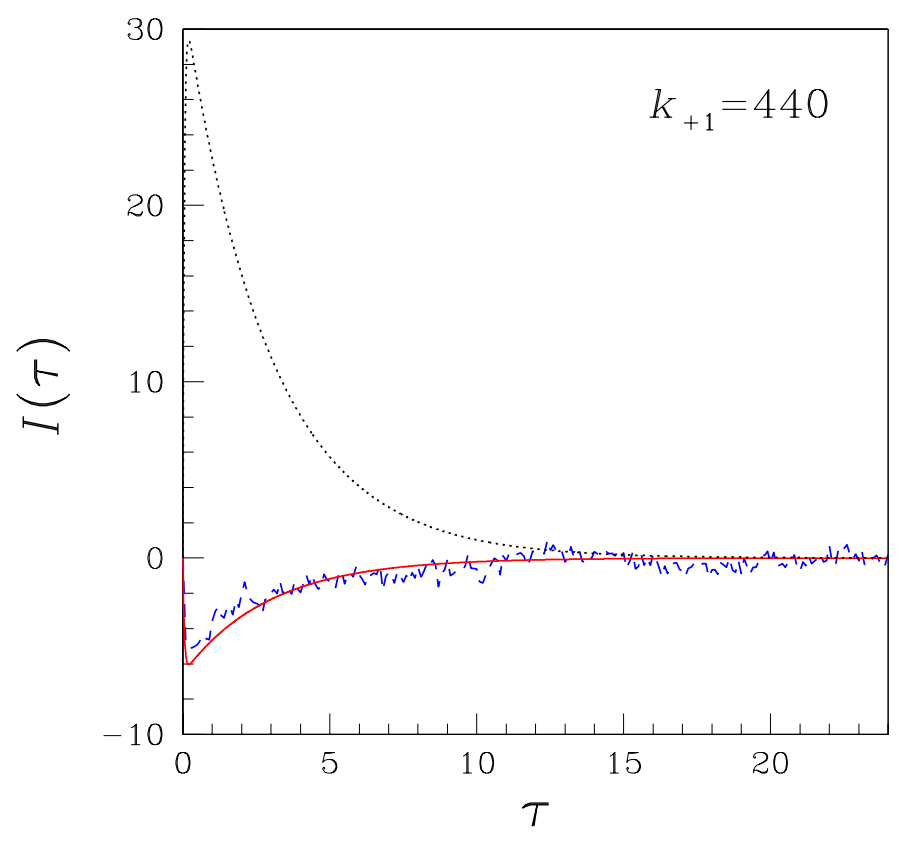

Figure 12. System with SNIPER bifurcation. Difference of temporal crosscorrelation functions $I(\tau)=\langle x(0) y(\tau)\rangle-\langle x(\tau) y(0)\rangle$ versus time lag $\tau$ for $k_{+1}=440$ above the saddle-node infinite period bifurcation. The black dotted line refers to the result of Eq. (13) deduced from Langevin approach to the system associated with linear deterministic dynamics. The red solid line refers to the result of Eq. (12) deduced from Langevin approach to the nonlinear mechanism given in Eqs. (66-69). The blue dashed line refers to the results of the direct simulation of the master equation associated with Eqs. (66-69). The other parameter values are given in the caption of Fig. 10. The statistics is performed over $5 \times 10^{5}$ stochastic trajectories.

Clearly, the behavior of the fluctuations is less and less correctly captured by a local stochastic analysis as the bifurcation gets closer.

\section{Discussion and Conclusion}

Time asymmetry of fluctuations observed in far-from-equilibrium systems [3] incited us to design a method to evaluate a reaction flux in a chemical or biological system with at least two variable concentrations. The difference $I(\tau)$ of time cross-correlations of concentration fluctuations can be experimentally determined in 


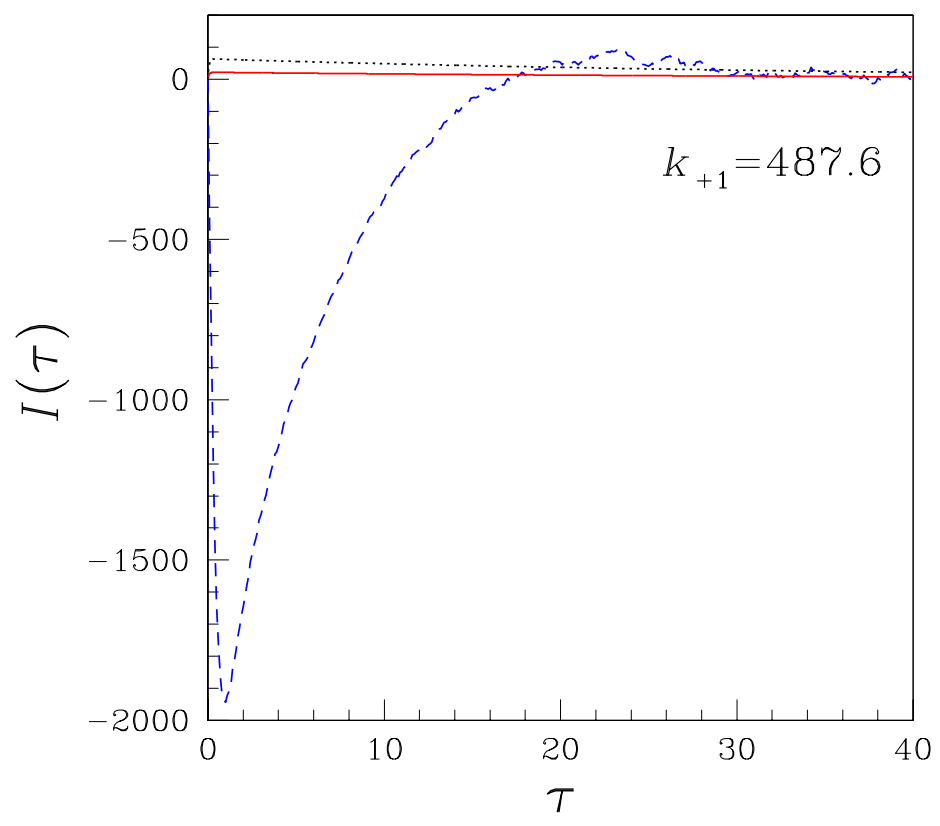

Figure 13. System with SNIPER bifurcation. Same caption as in Fig. 12 for $k_{+1}=487.6$, close to the saddle-node infinite period bifurcation.

small biological systems using, for example, tagged fluorescent species and fluctuation correlation spectroscopy (FCS) $[14,15]$. When the deterministic rate equations for concentrations are linear, the reaction flux can be deduced from the slope of $I(\tau)$ for vanishing time delay $\tau$, without any further knowledge of the system of interest [16].

We have determined approximate, analytical expressions of the difference of time cross-correlations of concentration fluctuations using Langevin-type equations associated with biologically relevant nonlinear chemical mechanisms. Systems exhibiting bistability and periodic oscillations of different natures have been studied. The results have been compared with simulations of the master equation, considered as a reference. For noncritical parameter values, the predictions of the Langevin approach to nonlinear dynamics perfectly agree with the results of the master equation.

However, discrepancies between the two approaches are observed in the vicin- 
ity of a bifurcation. Indeed, the Langevin equations are deduced from the master equation by a truncation of the Kramers-Moyal expansion to the two first terms, the drift and the diffusion term [20]. The Kramers-Moyal expansion results from a Taylor expansion of the master equation, when the stochastic processes are assumed to involve only small jumps of the random variables [20]. A bifurcation is often associated with vanishing real parts of the eigenvalues of the deterministic operator and, consequently, small real parts of the drift term. Hence, terms of order higher than two in the Kramers-Moyal expansion may be not negligible close to a bifurcation and Langevin approach may fail. This phenomenon is observed in the case of the Hopf bifurcation. In the case of the saddle-node infinite period bifurcation, another effect magnifies the discrepancies between the Langevin approach and the master equation. Actually, we use linearized Langevin equations around the steadystate whereas the SNIPER bifurcation would require a global analysis due to the emergence of a directly well-developed attractor.

In the generic case of noncritical parameter values, the estimation of a reaction flux from the determination of correlation function remains delicate if the chemical system of interest is entirely unknown. Indeed, the Langevin approach to nonlinear dynamics shows that the amplitude of $I(\tau)$ is proportional to the reaction flux but also to a specific combination of parameters which depends on the nonlinearities of dynamics. Assuming linear dynamics and applying the simple, general expression of cross-correlation functions obtained in this case often leads to mediocre estimations of the reaction flux. Even the sign of the flux may be wrong.

From a theoretical point of view, the interplay between fluctuations and nonlinearities of deterministic dynamics is subtle and leads to specific expressions for the time cross-correlations of concentration fluctuations, that depend on the details of the dynamics. In order to harvest the determination of correlation functions for reaction flux estimation in a given chemical system, it is therefore essential, first, 
to identify the reaction mechanism [34], then, to evaluate the associated rate constants [35], and, finally, to compute the specific expression of the difference of time cross-correlation functions for this mechanism. If these demanding requirements may be fulfilled and provided that the system is far from a bifurcation, the stochastic differential equations of Langevin type governing the fluctuating dynamics of concentrations provides a reliable, analytical formula relating the reaction flux and the correlations of fluctuations in the vicinity of a stable steady state.

Other attempts have been made to derive entropy production in driven systems without knowing dynamics in detail through the analysis of time reversal asymmetry of stationary time series corresponding to stochastic realisations of trajectories associated with the considered physical mechanism. Applications to flashing ratchets [36] and chemical systems [37] have been proposed but the estimation of entropy production has been shown to sensitively depend on the coarse graining of the stochastic trajectory [38]. Consequently, the quantitative determination of fluxes in nonequilibrium systems using the estimation of entropy production from stochastic time series without further information on the system remains challenging.

The fluctuation-dissipation theorem, which relates correlation functions of fluctuations of an observable and response function to a perturbation, has also been generalized to nonequilibrium systems [39, 40]. The modified fluctuation-dissipation theorem has been experimentally verified in the vicinity of a nonequilibrium steady state by studying the position of a colloidal particle confined in an optical trap [41]. The comparison between the correlations of fluctuations of particle position and the response function to a weak perturbation has been used to confirm the theoretical predictions. However, the method relies on the computation of the difference between the results of two series of independent experiments. In such conditions, the control of the experimental uncertainties is crucial and has been done carefully in a 
system designed to this purpose, with the possibility to test the expected behaviors. In the case of unknown systems, the implementation of a checking protocol is more difficult and the possibility to use the method in a blind manner is a delicate task.

In conclusion, the estimation of generalized fluxes in far-from-equilibrium systems remains a challenging issue.

\section{Acknowledgments}

We thank l'Agence Nationale de Recherche (ANR T-KiNet project) for support.

\section{References}

[1] A. Goldbeter, Biochemical oscillations and cellular rhythms (Cambridge, Cambridge) 1996 .

[2] J. D. Murray, Mathematical biology: I An introduction (Springer, New York) 2002.

[3] U. M. B. Marconi, A. Puglisi, L. Rondoni and A. Vulpiani, Phys. Rep. 461, 111 (2008).

[4] D. J. Evans, E. G. D. Cohen and G. P. Morriss, Phys. Rev. Lett. 71, 2401 (1993).

[5] G. Gallavotti and E. G. D. Cohen, J. Stat. Phys. 80, 931 (1995).

[6] D. J. Evans and D. J. Searles, Adv. Phys. 52, 1529 (2002).

[7] P. Gaspard J. Chem. Phys. 120, 8898 (2004).

[8] W. Min, L. Jiang, J Yu, S.C. Kou, H. Qian and X. S. Xie, Nano Lett. 5, 2373 (2005).

[9] H. Ge, M. Qian and H. Qian, Phys. Rep. 510, 87 (2012).

[10] C. Giberti, L. Rondoni, and C. Vernia, Physica D 228, 64 (2007). 
[11] C. Paneni, D. J. Searles and L. Rondoni, J. Chem. Phys. 128, 164515 (2008).

[12] D. J. Stephens and V. J. Allan, Science 300, 82 (2003).

[13] N. C. Shaner, P. A. Steinbach, and R. Y. Tsien, Nat. Meth. 2, 905 (2005).

[14] H. Qian and E. L. Elson, Proc. Natl. Acad. Sci. USA 101, 2828 (2004).

[15] D. R. Sisan, D. Yarar, C. M. Waterman and J. S. Urbach, Biophys. J. 98, 2432 (2010).

[16] W. J. Heuett and H. Qian, J. Chem. Phys. 124, 044110 (2006).

[17] S. R. de Groot and P. Mazur, Non-Equilibrium Thermodynamics (NorthHolland, Amsterdam) 1962.

[18] C. Bianca and A. Lemarchand, J. Chem. Phys. 140, 224105 (2014).

[19] C. Bianca and A. Lemarchand, J. Chem. Phys. 141, 144102 (2014).

[20] N. G. van Kampen, Stochastic processes in physics and chemistry (North Holland, Amsterdam) 1983.

[21] C. W. Gardiner, Handbook of Stochastic Methods (Springer, Berlin) 1985.

[22] D. T. Gillespie, J. Chem. Phys. 113, 297 (2000).

[23] A. K. Gustavsson, D. D. Niekerk, C. B. Adiels, B. Kooi, M. Goksör, and J. L. Snoep, FEBS J. 281, 2784 (2014).

[24] J. E. Ferrell Jr., J. R. Pomerening, S. Y. Kim, N. B. Trunnell, W. Xiong, C.-Y. F. Huang, and E. M. Machleder, FEBS Lett. 583, 3999 (2009).

[25] G. Nicolis and I. Prigogine, Self-Organization in Nonequilibrium Systems (Wiley, New York) 1977. 
[26] D. T. Gillespie, A. Hellander and L. R. Petzold, J. Chem. Phys. 138, 170901 (2013).

[27] P. Gaspard, J. Chem. Phys. 117, 8905 (2002).

[28] J. Guckenheimer and P. Holmes, Nonlinear oscillations, dynamical systems and bifurcations of vector fields (Springer, New York) 1983.

[29] S. H. Strogatz, Nonlinear dynamics and chaos: with applications to physics, biology, chemistry, and engineering (Addison-Wesley, Reading) 1994.

[30] V. I. Arnold, Supplementary chapters to the theory of ordinary differential equations (Nauka, Moscow) 1978.

[31] J. Tyson, A. Csikasz-Nagy and B. Nowak, BioEssays 24, 1095 (2002).

[32] A. Csikasz-Nagy, D. Battogtokh, K. C. Chen, B. Novak, J. J. Tyson Biophys. J. 90, 4361 (2006).

[33] R. Erban, S. J. Chapman, I. G. Kevrekidis and T. Vejchodsky, SIAM J. Appl. Math. 70, 984 (2009).

[34] A. Lemarchand, H. Berthoumieux, L. Jullien and C. Gosse, J. Phys. Chem. A116, 8455 (2012).

[35] F. Closa, C. Gosse, L. Jullien and A. Lemarchand, J. Chem. Phys. 138, 244109 (2013).

[36] É. Roldàn and J. M. R. Parrondo, Phys. Rev. Lett. 105, 150607 (2010).

[37] S. Muy, A. Kundu, and D. Lacoste, J. Chem. Phys. 139, 124109 (2013).

[38] M. Esposito, Phys. Rev. E 85, 041125 (2012).

[39] U. Seifert and T. Speck, Europhys. Lett. 89, 10007 (2010). 
[40] G. Verley, R. Chetrite, and D. Lacoste, J. Stat Mech. P10025 (2011).

[41] J. R. Gomez-Solano, A. Petrosyan, S. Ciliberto, R. Chetrite and K. Gawedzki, Phys. Rev. Lett. 103, 040601 (2009). 